\title{
Nanoscale multifunctional sensor formed by a Ni nanotube and a scanning Nb nanoSQUID
}

\author{
J. Nagel, ${ }^{1}$ A. Buchter, ${ }^{2}$ F. Xue (薛飞), ${ }^{2}$ O. F. Kieler, ${ }^{3}$ T. Weimann, ${ }^{3}$ J. Kohlmann, ${ }^{3}$ A. B. Zorin, ${ }^{3}$ D. Rüffer, ${ }^{4}$ \\ E. Russo-Averchi, ${ }^{4}$ R. Huber, ${ }^{5}$ P. Berberich, ${ }^{5}$ A. Fontcuberta i Morral,${ }^{4}$ D. Grundler,${ }^{5,6}$ R. Kleiner, ${ }^{1}$ D. Koelle, ${ }^{1, *}$ \\ M. Poggio, ${ }^{2}$ and M. Kemmler ${ }^{1}$ \\ ${ }^{1}$ Physikalisches Institut and Center for Collective Quantum Phenomena in LISA ${ }^{+}$, Universität Tübingen, Auf der Morgenstelle 14, D-72076 \\ Tübingen, Germany \\ ${ }^{2}$ Department of Physics, University of Basel, Klingelbergstrasse 82, CH-4056 Basel, Switzerland \\ ${ }^{3}$ Fachbereich 2.4 "Quantenelektronik", Physikalisch-Technische Bundesanstalt, Bundesallee 100, 38116 Braunschweig, Germany \\ ${ }^{4}$ Laboratoire des Matériaux Semiconducteurs, Institut des Matériaux, Ecole Polytechnique Fédérale de Lausanne, \\ CH-1015 Lausanne, Switzerland \\ ${ }^{5}$ Lehrstuhl für Physik funktionaler Schichtsysteme, Physik Department E10, Technische Universität München, 85747 Garching, Germany \\ ${ }^{6}$ Institut des Matériaux, Faculté Sciences et Technique de l'Ingénieur, Ecole Polytechnique Fédérale de Lausanne, 1015 Lausanne, Switzerland
}

(Received 28 February 2013; revised manuscript received 4 July 2013; published 29 August 2013)

\begin{abstract}
Nanoscale magnets might form the building blocks of next generation memories. To explore their functionality, magnetic sensing at the nanoscale is key. We present a multifunctional combination of a nanometer-sized superconducting quantum interference device (nanoSQUID) and a Ni nanotube attached to an ultrasoft cantilever as a magnetic tip. By scanning the $\mathrm{Nb}$ nanoSQUID with respect to the $\mathrm{Ni}$ tube, we map out and analyze their magnetic coupling, demonstrate the imaging of an Abrikosov vortex trapped in the SQUID structure - which is important in ruling out spurious magnetic signals — and reveal the high potential of the nanoSQUID as an ultrasensitive displacement detector. Our results open a new avenue for fundamental studies of nanoscale magnetism and superconductivity.
\end{abstract}

DOI: 10.1103/PhysRevB.88.064425

PACS number(s): 85.25.Dq, 74.78.Na, 68.37.Rt, 74.25.F-

\section{INTRODUCTION}

There is growing interest in the investigation of small spin systems, such as molecular magnets, ${ }^{1-3}$ single chain magnets, ${ }^{4}$ single electrons, ${ }^{5}$ or cold atom clouds. ${ }^{6}$ Various detection schemes, e.g., magnetooptical spin detection, ${ }^{7,8}$ magnetic resonance force microscopy, ${ }^{9}$ or scanning-tunneling-microscopy assisted electron spin resonance, ${ }^{10,11}$ have been developed to detect such systems. Unlike these techniques, superconducting quantum interference devices (SQUIDs) directly measure the stray magnetic flux produced by a small magnetic particle (SMP) with a large bandwidth. ${ }^{12,13}$ This capability is especially interesting for the study of SMPs that support magnetic states not normally allowed in macroscopic magnets. ${ }^{14-17}$

A direct current (dc) SQUID is a superconducting loop, intersected by two Josephson junctions, and works as a flux-to-voltage transducer, i.e., the magnetic flux $\Phi$ threading the loop modulates the voltage $V$ across the junctions, with a period of the magnetic flux quantum $\Phi_{0}=h / 2 e$ (see, e.g., Ref. 18). Since the magnetic field distribution of a SMP is very close to that of a magnetic dipole, the figure of merit for SQUIDs is the spin sensitivity $S_{\mu}^{1 / 2}=S_{\Phi}^{1 / 2} / \phi_{\mu}$. Here, $S_{\Phi}$ is the spectral density of flux noise power and $\phi_{\mu} \equiv \Phi / \mu$ is the coupling factor, i.e., the flux coupled to the SQUID per magnetic moment $\mu \equiv|\vec{\mu}|$ of the SMP. Both $S_{\Phi}$ and $\phi_{\mu}$ can be optimized by scaling the SQUID down to nanometer dimensions. ${ }^{19-22}$ Various fabrication techniques, e.g., electron-beam lithography, ${ }^{23,24}$ focused ion beam milling, ${ }^{22,25,26}$ atomic force microscopy anodization, ${ }^{27,28}$ selfaligned shadow evaporation, ${ }^{29}$ or a combination of electronbeam lithography with the use of carbon nanotube junctions, ${ }^{30}$ have been used to realize nanoSQUIDs.

The experimental determination of $S_{\Phi}$ poses no basic difficulties, in some cases yielding very low $S_{\Phi}^{1 / 2} \approx$
$0.2-0.3 \mu \Phi_{0} / \sqrt{\mathrm{Hz}}$ (Refs. 23,24,26, and 31). In contrast, the determination of $\phi_{\mu}$ is not straightforward, as it depends on the position $\vec{r}_{p}$ and orientation $\hat{e}_{\mu}$ of $\vec{\mu}$ relative to the SQUID loop and on the SQUID geometry. Up to now, $\phi_{\mu}$ has been estimated by numerical or analytical calculations, which often rely on strongly simplifying assumptions. ${ }^{20,21,32}$ Also, a more advanced routine for calculating $\phi_{\mu}\left(\hat{e}_{\mu}, \vec{r}_{p}\right)$, which takes explicitly into account the SQUID geometry, ${ }^{22,24}$ has not yet been validated experimentally.

Here, we present a multifunctional sensor system, which combines a low-temperature magnetic force microscope (LTMFM) using a Ni nanotube as a ferromagnetic tip and a $\mathrm{Nb}$ nanoSQUID, optimized for SMP detection. This system allows for magnetization measurements of nanoscaled magnetic samples using very different measuring principles. In the case of LTMFM, forces acting on the magnetic tip are detected, e.g., allowing for the imaging of Abrikosov vortices in superconductors. ${ }^{33,34}$ For the nanoSQUID, signals caused by the entrance of such vortices are indistinguishable from signals produced by a SMP. Therefore the in situ imaging of Abrikosov vortices is an important prerequisite for reliable nanoSQUID magnetometry. In the first part of the article, we identify the position of trapped flux in the superconducting lead of the nanoSQUID operated in high magnetic fields. In contrast to the LTMFM, the nanoSQUID directly measures the stray flux from the magnetic tip coupled to the SQUID loop. Therefore, in the second part, we present measurements of $\Phi(\vec{r})$ for the half space above the nanoSQUID by scanning a cantilever with a nanoscale ferromagnet at position $\vec{r}$ as a magnetic tip. These findings are not exclusive to the use of a $\mathrm{Ni}$ nanotube, but should be valid for a wide range of SMPs. Furthermore, we show that the nanoSQUID can be used as a highly sensitive detector of displacement of the Ni nanotube. 


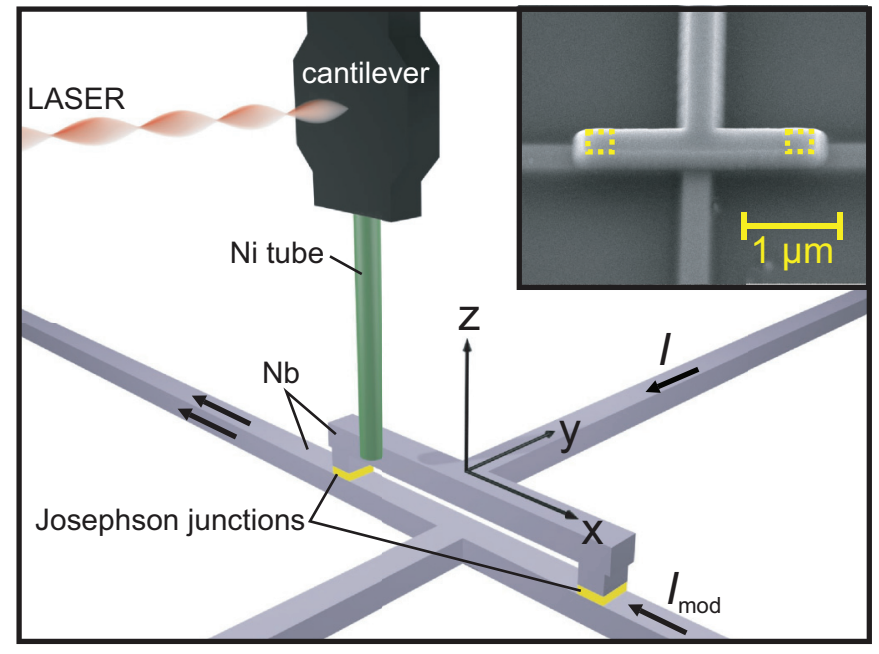

FIG. 1. (Color online) Schematic view (not to scale) of the nanoSQUID and Ni nanotube geometry, indicating $x, y, z$ directions as used below, with the origin centered on the surface of the upper $\mathrm{Nb}$ layer. Thick arrows indicate flow of applied bias current $I$ and modulation current $I_{\text {mod }}$. Inset shows scanning electron microscopy (SEM) image of the $\mathrm{Nb}$ nanoSQUID; dotted lines indicate the two Josephson junctions.

\section{SQUID LAYOUT, PROPERTIES, AND READOUT}

For the experiments presented here, we use a dc SQUID which has a sandwich-like geometry (see Fig. 1), i.e., the two arms of the SQUID loop lie directly on top of each other, and are connected via two $200 \times 200 \mathrm{~nm}^{2}$ planar $\mathrm{Nb} / \mathrm{HfTi} / \mathrm{Nb}$ Josephson junctions. ${ }^{35,36}$ The electric transport and noise properties of $\mathrm{Nb}$ nanoSQUIDs with this layout are described in Ref. 31. For this geometry, the size of the SQUID loop (in the $x-z$ plane) is given by the gap $(\sim 225 \mathrm{~nm})$ between the top and bottom $\mathrm{Nb}$ layers and the lateral distance $(\sim 1.8 \mu \mathrm{m})$ between the two junctions. Using such a geometry, a very small loop size, and hence a small loop inductance of a few $\mathrm{pH}$ or even lower can be achieved, which is essential for obtaining very low values for $S_{\Phi}$ (Ref. 37). The rms flux noise for the SQUID used here is $S_{\Phi}^{1 / 2} \approx 220 \mathrm{n} \Phi_{0} / \sqrt{\mathrm{Hz}}$ (in the white noise limit above $\sim 1 \mathrm{kHz}$ ). This value was measured in a separate, magnetically and electrically shielded setup, using a sensitive cryogenic amplifier for SQUID readout.

The nanoSQUID is mounted in a vacuum chamber (pressure $\leqslant 1 \times 10^{-6}$ mbar) at the bottom of a continuous-flow ${ }^{3} \mathrm{He}$ cryostat. The SQUID is biased at a current $I$ slightly above its critical current and at a magnetic flux $\Phi_{\text {mod }} \propto I_{\text {mod }}$ coupled via the modulation current $I_{\text {mod }}$ to the loop (cf. Fig. 1). To maintain operation of the SQUID at its optimum working point, i.e., at the maximum slope of its $V(\Phi)$ curve, we use a flux-locked loop (FLL) with a room-temperature voltage preamplifier. The FLL couples a feedback flux $\Phi_{f}=-\Phi$ to compensate for any flux signal $\Phi$. Using such a scheme, the output voltage $V_{\text {out }} \propto I_{\text {mod }}$ provided by the feedback loop is directly proportional to $\Phi$; in our case $V_{\text {out }} / \Phi=2.55 \mathrm{~V} / \Phi_{0}$.

\section{LTMFM SETUP}

The magnetic tip used in our LTMFM setup is a $\ell=$ $6-\mu \mathrm{m}$ long Ni nanotube which is fabricated by the atomic layer deposition of $\mathrm{Ni}$ and a $\sim 25$-nm thick $\mathrm{AlO}_{x}$ interlayer on a $75-\mathrm{nm}$ diameter GaAs nanowire. ${ }^{38}$ The outer diameter $D_{a}=190( \pm 35) \mathrm{nm}$ (Ref. 39), yielding a thickness $t=$ $32.5( \pm 17.5) \mathrm{nm}$ of the Ni layer and hence a volume of the $\mathrm{Ni}$ tube $V_{\mathrm{Ni}}=0.096( \pm 0.063) \mu \mathrm{m}^{3}$. The Ni nanotube is affixed parallel to the cantilever axis ( $z$ axis) such that it protrudes from the cantilever end by $4 \mu \mathrm{m}$. We define the position $\vec{r}=(x, y, z)$ of the Ni tip (relative to the SQUID) as the intersection point of its cylindrical axis with the bottom end of the tube. The cantilever hangs above the SQUID in the pendulum geometry, i.e., perpendicular to the scanned surface (in the $x-y$ plane; cf. Fig. 1). ${ }^{40}$ A three-dimensional (3D) piezoelectric positioning stage (Attocube Systems AG) moves the SQUID relative to the $\mathrm{Ni}$ nanomagnet. In noncontact scanning force microscopy, the above-described configuration prevents the tip of the cantilever from snapping into contact with the sample surface and thus allows for the use of particularly soft - and therefore sensitive - cantilevers (spring constant $\leqslant 1 \mathrm{mN} / \mathrm{m}$ ). Similar setups were used in experiments investigating noncontact friction between closely spaced bodies ${ }^{41}$ and ultrasensitive magnetic resonance force microscopy. ${ }^{42}$ The single-crystal Si cantilever used here is $120-\mu \mathrm{m}$ long, $4-\mu \mathrm{m}$ wide, and $0.1-\mu \mathrm{m}$ thick and includes a 15- $\mu \mathrm{m}$ long, $1-\mu \mathrm{m}$ thick mass on its end; for details see Ref. 43. The oscillation of the lever along the $y$ direction is detected using laser light focused onto a $10-\mu \mathrm{m}$ wide paddle near the mass-loaded end and reflected back into an optical fiber interferometer. ${ }^{44}$ One hundred $\mathrm{nW}$ of light are incident on the paddle from a temperature-tuned 1550-nm distributed feedback laser diode.

At temperature $T=4.3 \mathrm{~K}$ and applied magnetic field $H=$ 0 , the nanomagnet-loaded cantilever has a resonance frequency $f_{\text {res }}=3413 \mathrm{~Hz}$ and an intrinsic quality factor $Q_{0}=3.4 \times 10^{4}$. Its spring constant is determined to be $k=90 \mu \mathrm{N} / \mathrm{m}$ through measurements of its thermal noise spectrum at several different temperatures. As a result, far from the SQUID, where surface interactions do not play a role, ${ }^{41,45}$ the cantilever has a thermally limited force sensitivity of $10 \mathrm{aN} / \sqrt{\mathrm{Hz}}$. Note that under ambient conditions a MFM with such a soft cantilever, operated in the shear mode, high resolution images of the topography are hard to obtain. ${ }^{46}$ However, at very low temperature and pressure as used for the present work, the vibrating amplitudes of the cantilevers are very low. If the mean energy of the cantilever $k_{B} T$ ( $k_{B}$ is the Boltzmann constant) is converted to a mean displacement fluctuation $\left\langle y^{2}\right\rangle=k_{B} T / k$, we infer a displacement noise $<1 \mathrm{~nm} / \mathrm{Hz}^{1 / 2}$. The interferometric cantilever deflection signal is fed through a field programmable gate array (FPGA) (National Instruments) circuit back to a piezoelectric element which is mechanically coupled to the cantilever. In this way, it is possible to selfoscillate the cantilever at its fundamental resonance frequency and at a desired amplitude.

We produce noncontact force microscopy images by scanning (in the $x-y$ plane for fixed $z$ ) the position of the nanomagnet-tipped cantilever over the SQUID and simultaneously measuring the cantilever resonance frequency $f_{\text {res }}(x, y)$, which is proportional to the force gradient $\partial F_{y} / \partial y$ acting on the nanomagnet-tipped cantilever. Although no feedback is used to stabilize the cantilever position, the system is stable against mechanical drift of a few nanometers within 
times relevant to the measurements presented here. From such images we can identify the topography of the nanoSQUID, allowing us to precisely position the Ni nanotube with respect to the nanoSQUID. At the same time, due to the magnetization of the Ni nanotube tip, the images show features produced by the diamagnetic response of the superconductor.

\section{MFM IMAGING OF AN ABRIKOSOV VORTEX}

Prior to the measurements of the magnetic flux coupled by the Ni tip to the SQUID, we investigate a possible impact of an applied magnetic field on the nanoSQUID. In particular, Abrikosov vortices that may enter the superconducting areas are a severe problem for nanoSQUID magnetometry since such vortices (i) can degrade the SQUID performance and (ii) generate spurious magnetic signals and therefore mimic a magnetic behavior not related to the sample under investigation. In this section we show that with the presented sensor system an in situ detection of Abrikosov vortices is possible. $H$ is aligned along the $z$ direction, with a possible tilt of a few degrees. The trapping of a vortex appears as a voltage jump in the periodic $V(H)$ characteristics, when the SQUID voltage is measured directly, rather than using the FLL readout. Note that the SQUID voltage oscillates with increasing $H$ due to the nonperfect alignment of $H$ along the $z$ direction, i.e., $H$ has a finite in-plane component, which induces magnetic flux threading the SQUID loop. From the effective area of the SQUID and the oscillation period of $V(H)$, we estimate a tilt of the applied field of $\sim 2^{\circ}$ with respect to the $z$ axis. An example for a vortex trapping process is shown in Fig. 2(a), where a huge jump in $V(H)$ occurs near $\mu_{0} H=50 \mathrm{mT}$. This observation is consistent with a strong jump (decrease) in the critical current vs applied field near $\mu_{0} H=50 \mathrm{mT}$, which we

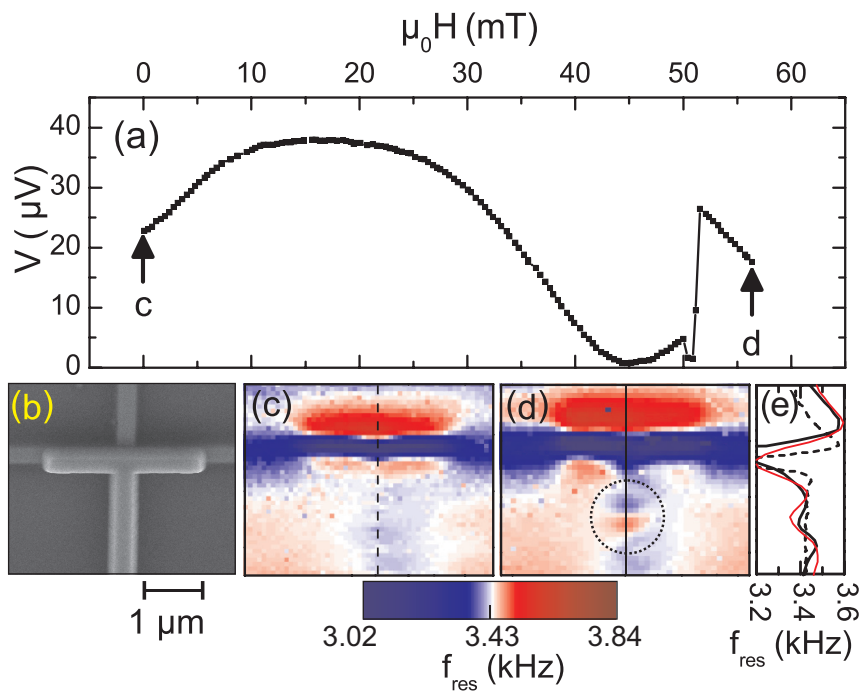

FIG. 2. (Color online) MFM imaging of trapped flux: (a) $V(H)$ for a single sweep from 0 to $56 \mathrm{mT}$; labels c, d indicate field values for LTMFM images shown in (c) and (d), respectively. (b) SEM image of the nanoSQUID. LTMFM images $f_{\text {res }}(x, y)$, (c) without trapped vortices at $H=0$ and (d) with a trapped vortex (indicated by dotted circle) at $\mu_{0} H=56 \mathrm{mT}$. (e) line scans along dashed line in (c) (dashed curve), solid line in (d) (solid curve) and calculated response for the SQUID with a trapped vortex along solid line in (d) (red curve). observed for another $\mathrm{Nb}$ nanoSQUID with the same layout. ${ }^{31}$ We note that during the field sweep, the Ni tip was retracted from the SQUID. For further improvement of the SQUID layout the knowledge of the position of trapped vortices is indispensable. The ability of the LTMFM setup to image stray fields can be used to visualize vortices in the superconductors as well as the magnetic field of the screening currents of the nanoSQUID itself. In Fig. 2(d), taken at a magnetic field above the jump in $V(H)$, such a vortex is visible in the superconducting lead (top Nb layer) of the nanoSQUID. The vortex appears as a distortion in the otherwise flat resonance frequency $f_{\text {res }}$ distribution along the $\mathrm{Nb}$ line [cf. line scans in Fig. 2(e)]. In the given setup $\Delta f_{\text {res }} \propto \partial^{2} B_{M} / \partial y^{2}$; here $B_{M}$ is the projection of the flux density along the magnetization axis of the Ni tube, which is very close to the $z$ axis. Hence, for an undisturbed vortex a symmetric tripolar response is expected. The solid red line in Fig. 2(e) shows a line scan along the solid line in Fig. 2(d) of the expected vortex signal calculated with 3D-MLSI. From this simulation, we identify the origin of the deviations from the expected symmetric tripolar signal to be the current distribution inside the superconducting structures. In contrast, at fields below the jump the trapped vortex is absent [see Fig. 2(c)]. For the subsequent investigations, we operate the SQUID in nominally zero field only, i.e., trapped vortices do not play a role.

\section{EXPERIMENTAL DETERMINATION OF $\Phi(\vec{r})$}

To determine $\Phi(\vec{r})$ we measure the nanoSQUID signal, i.e., the magnetic flux $\Phi$ through the SQUID loop as a function of the Ni nanotube position $(x, y)$ for fixed $z$. Such measurements produce images $\Phi(x, y)$ of the spatially dependent magnetic coupling of the Ni nanotube to the nanoSQUID. The experiment was performed in the following way: First, we bring the Ni nanotube into a well-defined saturated magnetic state along its easy axis. This is done by a half magnetization cycle, i.e., a sweep of $H$ (aligned along $z$ direction, as above) from zero to $\mu_{0} H_{\max }=-150 \mathrm{mT}$ and back to $H=0$. From previous experiments, we know that $H_{\max }$ is strong enough to saturate the magnetization of the Ni nanotube..$^{38,47}$ To avoid trapped flux in the SQUID, the magnetization cycle is performed at $T=14 \mathrm{~K}$, i.e., significantly above the transition temperature $T_{c} \sim 9 \mathrm{~K}$ of the Nb SQUID. Subsequently, we zero-field cool the SQUID to its operation temperature $T=4.3 \mathrm{~K}$, and then set up the FLL readout for the SQUID. Even for a worst case scenario, i.e., maximum modulation and screening currents, the resulting magnetic field applied to the nanotube by the SQUID currents is less than $5 \mathrm{mT}$, which is well below the coercive field of the nanotube. We therefore assume a fully saturated nanotube for subsequent results. For various distances $z$ between the tip and the top Nb layer of the SQUID we make scans in the $x-y$ plane with a scan range of about $6 \times 7 \mu \mathrm{m}^{2}$ corresponding to $81 \times 81$ pixels. The scans start at the largest distance of $z \approx 700 \mathrm{~nm}$. In steps of $50 \mathrm{~nm}$ the distance is subsequently reduced until the tip touches the top $\mathrm{Nb}$ layer of the SQUID (at $z=0$ ), which is detected as a loss of the oscillation of the cantilever. The touchpoint is also necessary for the calibration of the $z=0$ position.

Figures 3(a) and 3(b) shows two representative $\Phi(x, y)$ images taken at (a) $z=100 \mathrm{~nm}$ and (b) $z=710 \mathrm{~nm}$. The 

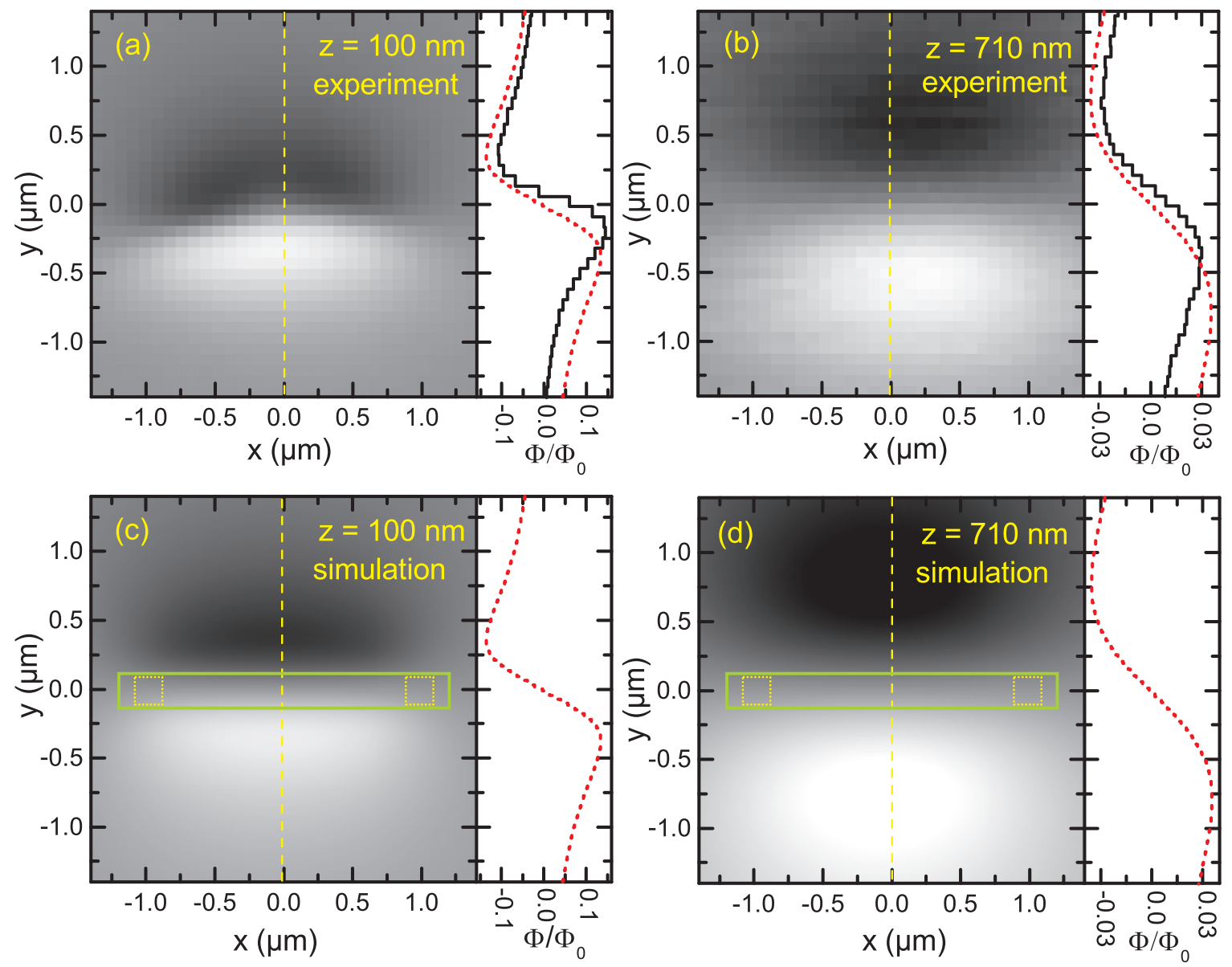

FIG. 3. (Color online) Magnetic flux $\Phi$ generated in the SQUID vs $x-y$ position of the Ni nanotube (magnetized along the $z$ axis). In (c) and (d), solid rectangle and dotted squares indicate position of the SQUID and the two junctions, respectively. Vertical dashed lines indicate position of line scans $\Phi(y)$ to the right of each image. (a, b) Upper graphs show experimental results and (c, d) lower graphs show corresponding simulation results, using Eq. (1) for $z=100 \mathrm{~nm}$ (left graphs) and $z=710 \mathrm{~nm}$ (right graphs). For the simulation we assumed $M_{s}=408 \mathrm{kA} / \mathrm{m}$ and $V_{\mathrm{Ni}}=0.047 \mu \mathrm{m}^{3}$. Linescans in (a,b) also include calculated line scans from (c,d).

images show a bipolar flux response, i.e., when the tip crosses the SQUID loop the flux signal is inverted. For the closer distance [Fig. 3(a)] the induced flux is stronger and spatially more confined as compared with the larger distance [Fig. 3(b)]. At $z=100 \mathrm{~nm}$, we obtain $\Delta \Phi=\Phi_{\max }-\Phi_{\min } \approx 0.26 \Phi_{0}$, with the positions of the maximum $\Phi_{\max }$ and minimum $\Phi_{\min }$ in the line scan $\Phi(y)($ at $x=0)$ being separated by $\Delta y=370 \mathrm{~nm}$. For $z=710 \mathrm{~nm}$, we find $\Delta \Phi \approx 0.06 \Phi_{0}$ and $\Delta y=750 \mathrm{~nm}$ [cf. black solid lines in the right panels in Figs. 3(a) and 3(b)].

\section{ANALYSIS WITH $\phi_{\mu}\left(\vec{r}_{p}\right)$}

To analyze the measured flux signals, we start from numerical simulations of $\phi_{\mu}\left(\hat{e}_{\mu}, \vec{r}_{p}\right)$ for a point-like SMP with orientation $\hat{e}_{\mu}$ of its magnetic moment at position $\vec{r}_{p}$ in the $3 \mathrm{D}$ space above the SQUID loop. ${ }^{22,24}$ This routine takes explicitly into account the geometry in the plane of the SQUID loop, and is based on the numerical simulation of the two-dimensional (2D) sheet current density in the SQUID loop, using London theory. ${ }^{49}$

Figure 4 shows the calculated coupling factor $\phi_{\mu}$ in the $y-z$ plane, with the SQUID loop in the $x-z$ plane and the magnetic

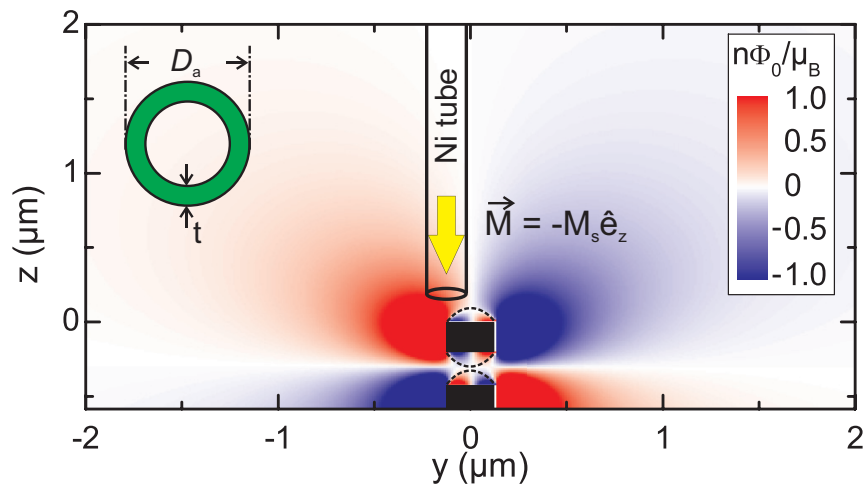

FIG. 4. (Color online) Calculated coupling factor $\phi_{\mu}$ in the $y$ $z$ plane $(x=0)$ for a point-like magnetic particle with magnetic moment $\vec{\mu}$ along $-\hat{e}_{z}$. Black rectangles indicate position of the $\mathrm{Nb}$ top and bottom layer; dotted lines include regions for which the simulations produce unphysical results (Ref. 48). A sketch of the bottom part of the Ni nanotube (drawn to scale) is shown within the coupling map to illustrate the spatial dependence of the coupling factor within the volume of the tube. Upper left inset schematically shows a zoomed cross section of the Ni nanotube. 
moment pointing along the $-z$ direction. $\phi_{\mu}$ decreases with increasing distance from the SQUID loop and inverts when crossing the SQUID loop. This spatial dependence has a strong impact on the magnetic flux $\Phi(\vec{r})$ which is coupled by a $\mathrm{Ni}$ nanotube (at position $\vec{r}$ ) with finite size into the SQUID. For the calculation of $\Phi(\vec{r})$ we integrate $\phi_{\mu}$ over the volume $V_{\mathrm{Ni}}$ of the Ni nanotube at position $\vec{r}$ and multiply this with the $\mathrm{Ni}$ saturation magnetization $M_{s}$, i.e.,

$$
\Phi(\vec{r})=M_{s} \int_{V_{\mathrm{Ni}}(\vec{r})} \phi_{\mu}\left(\vec{r}_{p}\right) d V,
$$

assuming a homogeneous $M_{s}$ over the entire volume of the $\mathrm{Ni}$ nanotube.

Figures 3(c) and 3(d) show flux images $\Phi(x, y)$ calculated from Eq. (1) for $z=100$ and $710 \mathrm{~nm}$, respectively, with a saturation magnetization $M_{s}$ along the $-z$ direction (cf. Fig. 4). The bipolar flux response and the positions of the minima $\Phi_{\min }$ and maxima $\Phi_{\max }$ in $\Phi(y)$ (for $x=0$ ) are reproduced well by the simulations [cf. dotted lines in the right panels in Figs. 3(c) and 3(d), which are also shown for comparison with the experimental data in the right panels of Figs. 3(a) and 3(b)].

For a quantitative analysis, Fig. 5 compares experimentally obtained $\Phi_{\min }$ and $\Phi_{\max }$ for all investigated distances to the simulated ones. From previous work on similar $\mathrm{Ni}$ nanotubes, ${ }^{47}$ we know that the saturation magnetization is equal within the experimental $20 \%$ error to the bulk value known from the literature $M_{s}=408 \mathrm{kA} / \mathrm{m}$ (Ref. 50). A much larger uncertainty in the absolute values for $\Phi$, calculated from Eq. (1), comes from the uncertainty in the volume $V_{\mathrm{Ni}}$ of the $\mathrm{Ni}$ tube due to the large margins for the the $\mathrm{Ni}$ thickness $t$ and hence the outer diameter $D_{a}$. Therefore, we fixed $M_{s}=408 \mathrm{kA} / \mathrm{m}$ and used $t$ as an adjustable parameter to obtain the best quantitative agreement between the experiment and calculation, which we obtained for $t=17.5 \mathrm{~nm}$, corresponding to $D_{a}=160 \mathrm{~nm}$ and $V_{\mathrm{Ni}}=0.047 \mu \mathrm{m}^{3}$. This value is significantly smaller than the mean value for $V_{\mathrm{Ni}}$ of other Ni tubes from the same batch as quoted above. However, it is still within the large uncertainty for $V_{\mathrm{Ni}}$. Furthermore, an independent determination of $V_{\mathrm{Ni}}$ on the same $\mathrm{Ni}$ tube, via cantilever magnetometry, yields a value which is even somewhat below the one obtained via SQUID measurement. We note that the formation of a multidomain state close to the bottom of the Ni tube is unlikely, as hysteresis curves $M(H)$ measured both with SQUID and cantilever magnetometry

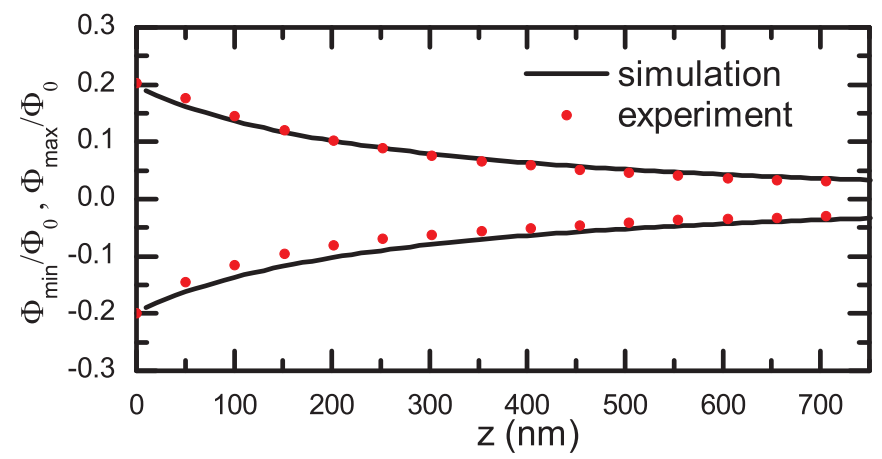

FIG. 5. (Color online) Experimental and simulated minimum and maximum flux signals, $\Phi_{\min }$ and $\Phi_{\max }$, versus distance $z$. For the simulation we assumed $M_{s}=408 \mathrm{kA} / \mathrm{m}$ and $V_{\mathrm{Ni}}=0.047 \mu \mathrm{m}^{3}$. indicate no reduction of the magnetic signal upon sweeping $H$ from $\pm H_{\text {max }}$ back to zero. In the experiment we also find an asymmetry in $\Phi(y)$, i.e., $\Phi_{\max } \geqslant\left|\Phi_{\min }\right|$. This effect is most likely caused by flux focusing effects of the feed lines in the top and bottom $\mathrm{Nb}$ layers, which are not considered in the simulations. The flux focusing effects are also visible in the distorted flux image (broken horizontal symmetry) in Fig. 3(a). An additional asymmetry may be caused by a slightly tilted tube with respect to the SQUID plane. In our case, however, this effect is considered to be small since the tilt angle is measured to be less than $5^{\circ}$. To conclude this section, we note that the measured flux coupled from the Ni tube to the nanoSQUID confirms our simulation routine for the coupling factor $\phi_{\mu}$ within the experimental error of $\sim 2$, which is due to the relatively large uncertainty in the thickness of the Ni tube.

\section{DISPLACEMENT DETECTION}

Finally, we discuss the sensitivity of our setup for the detection of the oscillatory motion of the cantilever by the SQUID. ${ }^{51,52}$ While the absolute flux signal from the $\mathrm{Ni}$ nanotube is optimally detected at the positions yielding $\Phi_{\max }$ and $\Phi_{\min }$, for the cantilever displacement detection, a large gradient $\partial \Phi / \partial y$ is required. The line scans in Fig. 3 clearly show that the optimum position for displacement detection is directly above the SQUID. For our device, we find for $z=50 \mathrm{~nm}$ a gradient $\Phi_{y} \equiv \partial \Phi / \partial y=2 \times 10^{6} \Phi_{0} / \mathrm{m}$. With the flux noise $S_{\Phi}^{1 / 2}=220 \mathrm{n} \Phi_{0} / \sqrt{\mathrm{Hz}}$, this yields an extremely low value for the predicted displacement sensitivity $S_{r}^{1 / 2}=$ $S_{\Phi}^{1 / 2} / \Phi_{y}=110 \mathrm{fm} / \sqrt{\mathrm{Hz}}$, which is already a factor of 2 below the best value found in the literature. ${ }^{51-53}$ Still, $S_{r}$ is by far not optimized and could be further improved by using a reduced linewidth for the SQUID arm in the top $\mathrm{Nb}$ layer and by increasing the number of spins in the magnet.

\section{CONCLUSION}

In conclusion, we experimentally determined the spatial dependence of the magnetic coupling between a Ni nanotube and a $\mathrm{Nb}$ nanoSQUID. Operating the nanoSQUID in a flux locked loop, we measured the flux through the SQUID loop $\Phi(\vec{r})$ generated by the $\mathrm{Ni}$ nanotube during the scan of the tip in 3D space above the nanoSQUID. This yields experimental information on the magnetic coupling factor $\phi_{\mu}$, which together with the flux sensitivity determines the spin sensitivity as the figure of merit for small magnetic particle detection by a nanoSQUID. Our results are in good agreement with a recently developed routine for numerical calculation of the coupling factor between a small magnetic particle and a nanoSQUID. This provides an important step toward the development of optimized nanoSQUIDs for the investigation of small magnetic particles. With the presented measurement system, we demonstrate a reliable and nondestructive in situ tool for the challenging task of positioning a nanoscaled magnet to the position of highest coupling of a nanoSQUID. Furthermore, with a proper readout technique, our highly flux-sensitive nanoSQUID can be used for displacement detection of the cantilever in an MFM with extremely good displacement sensitivity, which still can be further improved. By using an MFM imaging mode, we also 
demonstrate the imaging of Abrikosov vortices, which are trapped at high magnetic fields in the superconducting leads of the nanoSQUID. This technique is not only useful for the improvement of the high-field suitability of nanoSQUIDs, but even more importantly allows for the in situ differentiation between a signal originating from a SMP and a signal due to the entrance of a spurious Abrikosov vortex. Finally, we demonstrate the use of a nanoSQUID as a local probe of the stray fields produced by the $\mathrm{Ni}$ nanotube, which may be of great importance in understanding magnetization reversal in these magnetic nanostructures. ${ }^{54}$ Such investigations will be the subject of future work as will investigations of other SMPs.

\section{ACKNOWLEDGMENTS}

J. Nagel acknowledges support by the Carl-Zeiss-Stiftung. This work was funded by the Deutsche Forschungsgemeinschaft (DFG) via the SFB/TRR 21, by the European Research Council via ERC advanced grant SOCATHES, by the Canton Aargau, and by the Swiss National Science Foundation (SNF, Grant No. 200020-140478). MP, ER, and AFiM acknowledge support from the NCCR QSIT. The research leading to these results has received funding from the European Community's Seventh Framework Programme (FP7/2007-2013) under Grant Agreement No. 228673 MAGNONICS. *koelle@uni-tuebingen.de

${ }^{1}$ L. Thomas, F. Lionti, R. Ballou, D. Gatteschi, R. Sessoli, and B. Barbara, Nature (London) 383, 145 (1996).

${ }^{2}$ D. Gatteschi and R. Sessoli, Angew. Chem., Int. Ed. 42, 268 (2003).

${ }^{3}$ L. Bogani and W. Wernsdorfer, Nat. Mater. 7, 179 (2008).

${ }^{4}$ L. Bogani, A. Vindigni, R. Sessoli, and D. Gatteschi, J. Mater. Chem. 18, 4750 (2008).

${ }^{5}$ P. Bushev, D. Bothner, J. Nagel, M. Kemmler, K. B. Konovalenko, A. Loerincz, K. Ilin, M. Siegel, D. Koelle, R. Kleiner, and F. Schmidt-Kaler, Eur. Phys. J. D 63, 9 (2011).

${ }^{6}$ J. Fortágh and C. Zimmermann, Science 307, 860 (2005).

${ }^{7}$ J. R. Maze, P. L. Stanwix, J. S. Hodges, S. Hong, J. M. Taylor, P. Cappellaro, L. Jiang, M. V. Gurudev Dutt, E. Togan, A. S. Zibrov, A. Yacoby, R. L. Walsworth, and M. D. Lukin, Nature (London) 455, 644 (2008).

${ }^{8}$ G. Balasubramanian, I. Y. Chan, R. Kolesov, M. Al-Hmoud, J. Tisler, C. Shin, C. Kim, A. Wojcik, P. R. Hemmer, A. Krueger, T. Hanke, A. Leitenstorfer, R. Bratschitsch, F. Jelezko, and J. Wrachtrup, Nature (London) 455, 648 (2008).

${ }^{9}$ D. Rugar, R. Budakian, H. J. Mamin, and B. W. Chui, Nature (London) 430, 329 (2004).

${ }^{10}$ Y. Manassen, R. J. Hamers, J. E. Demuth, and A. J. Castellano, Jr., Phys. Rev. Lett. 62, 2531 (1989).

${ }^{11}$ C. Durkan and M. E. Welland, Appl. Phys. Lett. 80, 458 (2002).

${ }^{12}$ W. Wernsdorfer, Adv. Chem. Phys. 118, 99 (2001).

${ }^{13}$ J. Gallop, Supercond. Sci. Technol. 16, 1575 (2003).

${ }^{14}$ Z. K. Wang, M. H. Kuok, S. C. Ng, D. J. Lockwood, M. G. Cottam, K. Nielsch, R. B. Wehrspohn, and U. Gösele, Phys. Rev. Lett. 89, 027201 (2002).

${ }^{15}$ J. Topp, J. Podbielski, D. Heitmann, and D. Grundler, Phys. Rev. B 78, 024431 (2008).

${ }^{16}$ R. Streubel, D. J. Thurmer, D. Makarov, F. Kronast, T. Kosub, V. Kravchuk, D. D. Sheka, Y. Gaididei, R. Schäfer, and O. G. Schmidt, Nano Lett. 12, 3961 (2012).

${ }^{17}$ R. Streubel, V. P. Kravchuk, D. D. Sheka, D. Makarov, F. Kronast, O. G. Schmidt, and Y. Gaididei, Appl. Phys. Lett. 101, 132419 (2012).

${ }^{18}$ R. Kleiner, D. Koelle, F. Ludwig, and J. Clarke, Proc. IEEE 92, 1534 (2004).

${ }^{19}$ C. P. Foley and H. Hilgenkamp, Supercond. Sci. Technol. 22, 064001 (2009).

${ }^{20}$ M. Ketchen, D. Awschalom, W. Gallagher, A. Kleinsasser, R. Sandstrom, J. Rozen, and B. Bumble, IEEE Trans. Magn. 25, 1212 (1989).
${ }^{21}$ V. Bouchiat, Supercond. Sci. Technol. 22, 064002 (2009).

${ }^{22}$ J. Nagel, K. B. Konovalenko, M. Kemmler, M. Turad, R. Werner, E. Kleisz, S. Menzel, R. Klingeler, B. Büchner, R. Kleiner, and D. Koelle, Supercond. Sci. Technol. 24, 015015 (2011).

${ }^{23}$ R. F. Voss, R. B. Laibowitz, and A. N. Broers, Appl. Phys. Lett. 37, 656 (1980).

${ }^{24}$ J. Nagel, O. F. Kieler, T. Weimann, R. Wölbing, J. Kohlmann, A. B. Zorin, R. Kleiner, D. Koelle, and M. Kemmler, Appl. Phys. Lett. 99, 032506 (2011)

${ }^{25}$ A. G. P. Troeman, H. Derking, B. Borger, J. Pleikies, D. Veldhuis, and H. Hilgenkamp, Nano Lett. 7, 2152 (2007).

${ }^{26}$ L. Hao, J. C. Macfarlane, J. C. Gallop, D. Cox, J. Beyer, D. Drung, and T. Schurig, Appl. Phys. Lett. 92, 192507 (2008).

${ }^{27}$ V. Bouchiat, M. Faucher, C. Thirion, W. Wernsdorfer, T. Fournier, and B. Pannetier, Appl. Phys. Lett. 79, 123 (2001).

${ }^{28}$ M. Faucher, P.-O. Jubert, O. Fruchart, W. Wernsdorfer, and V. Bouchiat, Supercond. Sci. Technol. 22, 064010 (2009).

${ }^{29}$ A. Finkler, Y. Segev, Y. Myasoedov, M. L. Rappaport, L. Ne'eman, D. Vasyukov, E. Zeldov, M. E. Huber, J. Martin, and A. Yacoby, Nano Lett. 10, 1046 (2010)

${ }^{30}$ J.-P. Cleuziou, W. Wernsdorfer, V. Bouchiat, T. Ondarcuhu, and M. Monthioux, Nat. Nanotech. 1, 53 (2006).

${ }^{31}$ R. Wölbing, J. Nagel, T. Schwarz, O. Kieler, T. Weimann, J. Kohlmann, A. B. Zorin, M. Kemmler, R. Kleiner, and D. Koelle, Appl. Phys. Lett. 102, 192601 (2013).

${ }^{32}$ C. Granata, A. Vettoliere, R. Russo, E. Esposito, M. Russo, and B. Ruggiero, Appl. Phys. Lett. 94, 062503 (2009).

${ }^{33}$ A. Moser, H. J. Hug, I. Parashikov, B. Stiefel, O. Fritz, H. Thomas, A. Baratoff, H.-J. Güntherodt, and P. Chaudhari, Phys. Rev. Lett. 74, 1847 (1995).

${ }^{34}$ A. Volodin, K. Temst, C. V. Haesendonck, Y. Bruynseraede, M. I. Montero, and I. K. Schuller, Europhys. Lett. 58, 582 (2002).

${ }^{35}$ D. Hagedorn, R. Dolata, F.-I. Buchholz, and J. Niemeyer, Physica C 372-376, 7 (2002).

${ }^{36}$ D. Hagedorn, O. Kieler, R. Dolata, R. Behr, F. Müller, J. Kohlmann, and J. Niemeyer, Supercond. Sci. Technol. 19, 294 (2006).

${ }^{37}$ D. J. Van Harlingen, R. H. Koch, and J. Clarke, Appl. Phys. Lett. 41, 197 (1982).

${ }^{38}$ D. Rüffer, R. Huber, P. Berberich, S. Albert, E. Russo-Averchi, M. Heiss, J. Arbiol, A. Fontcuberta i Morral, and D. Grundler, Nanoscale 4, 4989 (2012). 
${ }^{39}$ The determination of $D_{a}$ for the tube investigated here was not possible, as the tube was lost during warm up after our measurements. Instead, we determined $D_{a}$ for six other tubes from the same batch by SEM. Those had values of $D_{a}$ varying from 155$225 \mathrm{~nm}$ with a variation in $D_{a}$ of $10-20 \mathrm{~nm}$ along a single tube.

${ }^{40}$ U. Gysin, S. Rast, M. Kisiel, C. Werle, and E. Meyer, Rev. Sci. Instrum. 82, 023705 (2011).

${ }^{41}$ B. C. Stipe, H. J. Mamin, T. D. Stowe, T. W. Kenny, and D. Rugar, Phys. Rev. Lett. 87, 096801 (2001).

${ }^{42}$ H. J. Mamin, R. Budakian, B. W. Chui, and D. Rugar, Phys. Rev. Lett. 91, 207604 (2003).

${ }^{43}$ B. W. Chui, Y. Hishinuma, R. Budakian, H. I. Mamin, T. W. Kenny, and D. Rugar, 12th International Conference on Solid-State Sensors, Actuators and Microsystems (Transducers '03), Vol. 2 (IEEE, Piscataway, NJ, 2003), pp. 1120-1123.

${ }^{44}$ D. Rugar, H. J. Mamin, and P. Guenther, Appl. Phys. Lett. 55, 2588 (1989).

${ }^{45}$ S. Kuehn, R. F. Loring, and J. A. Marohn, Phys. Rev. Lett. 96, 156103 (2006).

${ }^{46}$ K. Kim, Y. Seo, H. Jang, S. Chang, M.-H. Hong, and W. Jhe, Nanotechnology 17, S201 (2006).

${ }^{47}$ D. P. Weber, D. Rüffer, A. Buchter, F. Xue, E. Russo-Averchi, R. Huber, P. Berberich, J. Arbiol, A. Fontcuberta i Morral, D. Grundler, and M. Poggio, Nano Lett. 12, 6139 (2012).
${ }^{48}$ For simulating the current distribution, we have to assume that the currents are flowing in surface sheets rather than across the entire width of the two $\mathrm{Nb}$ lines (in the $y$ direction). This assumption allows for coupling of flux between the surface sheets, which produces the localized minima and maxima very close to the two SQUID arms, as visible in Fig. 4.

${ }^{49}$ M. M. Khapaev, A. Y. Kidiyarova-Shevchenko, P. Magnelind, and M. Y. Kupriyanov, IEEE Trans. Appl. Supercond. 11, 1090 (2001).

${ }^{50}$ C. Kittel, Introduction to Solid State Physics, 8th edition (Wiley and Sons, New York, 2005), p. 50.

${ }^{51}$ O. Usenko, A. Vinante, G. Wijts, and T. H. Oosterkamp, Appl. Phys. Lett. 98, 133105 (2011)

${ }^{52}$ A. Vinante, G. Wijts, O. Usenko, L. Schinkelshoek, and T. Oosterkamp, Nat. Commun. 2, 572 (2011).

${ }^{53}$ A. Vinante, A. Kirste, A. den Haan, O. Usenko, G. Wijts, E. Jeffrey, P. Sonin, D. Bouwmeester, and T. H. Oosterkamp, Appl. Phys. Lett. 101, 123101 (2012).

${ }^{54}$ A. Buchter, J. Nagel, D. Rüffer, F. Xue, D. P. Weber, O. F. Kieler, T. Weimann, J. Kohlmann, A. B. Zorin, E. Russo-Averchi, R. Huber, P. Berberich, A. Fontcuberta i Morral, M. Kemmler, R. Kleiner, D. Koelle, D. Grundler, and M. Poggio, Phys. Rev. Lett. 111, 067202 (2013). 